\title{
Human-Centered Design and Evaluation of Haptic Cueing for Teleoperation of Multiple Mobile Robots
}

\author{
Hyoung Il Son, Member, IEEE, Antonio Franchi, Member, IEEE, Lewis L. Chuang, Junsuk Kim, \\ Heinrich H. Bülthoff, Member, IEEE, and Paolo Robuffo Giordano, Member, IEEE
}

\begin{abstract}
In this paper, we investigate the effect of haptic cueing on human operator's performance in the field of bilateral teleoperation of multiple mobile robots, in particular multiple unmanned aerial vehicles (UAVs). Two aspects of human performance are deemed important in this area, namely the maneuverability of mobile robots and perceptual sensitivity of the remote environment. We introduce metrics that allow us to address these aspects in two psychophysical studies, which are reported here. Three fundamental haptic cue types were evaluated. The Force cue conveys information on the proximity of the commanded trajectory to obstacles in the remote environment. The Velocity cue represents the mismatch between the commanded and actual velocity of the UAVs and can implicitly provide a rich amount of information regarding the actual behavior of the UAVs. Finally, the Velocity+Force cue is a linear combination of the two. Our experimental results show that while maneuverability is best supported by the Force cue feedback, perceptual sensitivity is best served by the Velocity cue feedback. In addition, we show that large gains in the haptic feedbacks do not always guarantee an enhancement in teleoperator's performance.
\end{abstract}

Index Terms-Bilateral Teleoperation, Maneuverability, MultiRobot Systems, Perception, Psychophysics.

\section{INTRODUCTION}

$\mathbf{T}$ HE use of multi-robot systems, when compared to single robot approaches, allows several improvements to be achieved in terms reduced completion times and increased robustness [1], [2]. Given their high motion flexibility, unmanned aerial vehicles (UAVs) has been especially popular among the different multi-robot systems [3]. Recent years have seen

Manuscript received July 19, 2011 and revised December 9, 2011 and April 4, 2012. This paper was presented in part at the World Haptics Conference, Istanbul, Turkey, June 22-24, 2011, and in part at the International Conference on Intelligent Robots and Systems, San Francisco, California, September 2530, 2011. This research was supported in part by the Max Planck Society, the WCU (World Class University) program funded by the Ministry of Education, Science and Technology through the National Research Foundation of Korea (R31-10008), and a Korea Research Foundation grant funded by the Korean government (NRF-2011-357-D00003).

H. I. Son, A. Franchi, L. L. Chuang, and P. Robuffo Giordano are with the Max Planck Institute for Biological Cybernetics, Spemannstraße 38, 72076 Tübingen, Germany. E-mail: \{hyoungil.son, antonio.franchi, lewis.chuang, prg\}@tuebingen.mpg.de. Phone: +49-7071-601-218, Fax: +49-7071-601-616.

J. Kim is with the Department of Brain and Cognitive Engineering, Korea University, Anam-dong, Seongbuk-gu, Seoul, 136-713 Korea. E-mail: wowkjs@korea.ac.kr.

H. H. Bülthoff is with the Max Planck Institute for Biological Cybernetics, Spemannstraße 38, 72076 Tübingen, Germany, and with the Department of Brain and Cognitive Engineering, Korea University, Anam-dong, Seongbukgu, Seoul, 136-713 Korea. E-mail: hhb@tuebingen.mpg.de. an increasing development in the bilateral teleoperation algorithms for the efficient and robust control of multiple mobile robots (e.g., see [4], [5], [6], [7]). Nonetheless, comparatively less attention has been devoted towards understanding how teleoperation performance can be improved with regards to the human operator. Given this level of interest in multiplemobile-robot teleoperation, we believe that it is worthwhile to investigate how human control performance can be assessed within this context.

The overall performance of bilateral teleoperation systems relies on the human operator's command. Therefore, it seems advisable to take human control effort into consideration when defining performance metrics for our teleoperation system. For example, maneuverability performance would be considerably enhanced if the same tracking performance could be achieved with less control effort. This perspective seems especially reasonable in the case of a multi-robot system which can typically require more control effort for realizing an effective maneuvering when compared to the single-robot case, due to their more complex dynamics and interactions.

Similarly, a sensitive perception of the remote environment is often considered to be important for effective bilateral teleoperation systems. In the case of remote mobile robots (e.g., teleoperation of UAVs), visual feedback can often be limited by the on-board camera restrictions (e.g., restricted field-of-view, poor resolution) [8], [9]. This could severely limit teleoperational success and result in undesired collisions with remote obstacles. Providing haptic cues could alleviate this limitation. Unlike conventional teleoperation [10] (e.g., telesurgery), however, hard contact between flying UAVs and their physical environment is especially undesirable because they could cause severe damages to the mechanical structure of the robots. Therefore, there are no actual physical forces to be transmitted to the multi-UAV teleoperator and the conventional objectives of force tracking are not applicable. For this reason, pure force tracking may not be a measurable concept that can be directly generalized to the teleoperation of mobile robots. In this sense, transparency [11] is also not a suitable measure for the perceptual ability in the teleoperation of remote UAVs.

To sum up, evaluating human performance in the bilateral teleoperation of multi-UAVs should address the control effort required in maneuvering the mobile robots as well as the perceptual sensitivity of the operator of the remote environment. The contribution of providing haptic cues should be assessed accordingly. 


\section{A. Previous Work}

Frequency response analysis using transfer functions is a general approach in analyzing performance in the domain of bilateral teleoperation (e.g., transparency in [12], and position and force tracking in [13]). Likewise, the relationship between the teleoperator's control effort and maneuvering accuracy can be analyzed in terms of a frequency response function for the evaluation of maneuverability performance. Maneuverability has been proposed to be an important measure of the effectiveness of master-slave systems [14]. However, this work specifically focused on the transmission of position and force errors between the master and slave devices and not on actual human performance per se.

Some researchers have explicitly studied human performance in teleoperation systems. Chen et al. [15] provide excellent surveys on teleoperation systems in the context of human performance issues and user interface designs. Notably, maneuverability of a master haptic device has been studied in [16]. Here, the authors proposed a measure for the maneuverability of the master by considering the musculo-skeletal model of operator. Recently, Nambi et al. also studied the human controllability on the master device to see an effect of velocity, admittance gain and force on the performance using admittance-type devices [17].

Some research studies have addressed how human perceptual sensitivity can be enhanced in the conventional teleoperation [18], [19], [20], [21], [22]. Among these, fidelity has been proposed as an objective based on the claim that the information about the relative changes of environment impedance is more important for interactions with soft tissues than the environment impedance alone, see [18]. Taking inspiration from [18], Germsem et al. provided empirical evidence that a relative change in the transmitted stiffness to the operator could be increased by tuning the control parameters of a teleoperation controller [19]. Modified position/force scaling approaches were developed between the master and the slave, using a nonlinear filtered scaling and a time-varying scaling in [20] and [21] respectively. Son et al. proposed in [22] a quantitative index for the perception based on psychophysics and then showed that the operator's detection and discrimination could be enhanced by a perception-index-maximizedcontroller via psychophysical experiments.

In a recent study, the effectiveness of various artificial forces for collision avoidance was evaluated in the teleoperation of a single UAV [23]. An ecological interface paradigm was presented in [24], which provides multi-visual information into a 3D mixed-reality display in mobile robot teleoperation. A series of principles for presenting the information to improve an operator's situation awareness of a remote robot were presented in [24]. In addition, the effects of various factors (e.g., time delay, force-reflecting method, image-display alternatives, etc.) on obstacle avoidance performance of mobile robot teleoperation were studied through subjective tests using several measures [25]. A fuzzy force-reflection method was also proposed and compared against a conventional proportionalderivative (PD) type force-reflection method. There were more fundamental studies to understand human's neuromuscular system in a car driving with haptic feedback. Venrooij et al. proposed a method to measure biodynamic feedthrough and admittance of human operator simultaneously in a motion simulator [26]. A similar study was presented in [27] to determine a driver's biomechanical properties of the anklefoot complex during car following task.

Although it is evident that, recently, a number of researchers focus their attention on human perspective studies in mobile robot teleoperation, still, few works have addressed the case of teleoperation of multiple mobile robots.

\section{B. Objective and Outline}

The aim of the present paper is to build upon the control framework introduced in [6] by the authors and to discuss how information from the remote UAV group (slave-side) can be appropriately transformed into haptic cues for the human operator via the master device. Subsequently, we will present and discuss a systematic evaluation of the contribution of the proposed cues to teleoperation performance from the human operator perspective via psychophysical studies. To this end, we first introduce a performance index of the teleoperation system of remote UAVs, maneuverability, in terms of how easily the operator can control the movement of the slave system accurately. We then discuss a second performance index, perceptual sensitivity, aimed at measuring how well the operator is able to perceive the current state of the UAVs and their surrounding obstacles.

The structure of this paper is as follows. In Section II the concepts of maneuverability and operator perceptual sensitivity are introduced together with suitable metrics that take human performance into account. Following this, Section III reviews the control architecture for the bilateral teleoperation of multiple UAVs in [6]. Three haptic feedback algorithms are then introduced within the context of this framework. After presenting our experimental method in Section IV, these haptic feedback algorithms are subsequently evaluated, using our proposed metrics, to determine the algorithm that achieves the best level of maneuverability performance and operator sensitivity in Section V and VI, respectively, followed by discussions in Section VII. This paper ends with our conclusions and recommendations for future work.

\section{Performance MeAsures}

\section{A. Maneuverability}

1) Position tracking of slave robot: In conventional teleoperation research, the position tracking ability of the slave is typically evaluated by estimating the discrepancy between the positions of the slave and the master. In the current work, we are concerned with the discrepancy between the position of the slave and the target position on the reference path that the teleoperator intends to maneuver to. Therefore, position tracking error is defined as

$$
e_{p}(t):=\bar{x}(t)-x_{r e f}(t)
$$

for $N$ slave robots, wherein $\bar{x}(t)=\frac{1}{N} \sum_{i=1}^{N} x_{i}(t), x_{i}(t) \in \mathbb{R}^{3}$ represents the position of $i^{t h}$ robot and $x_{r e f}(t) \in \mathbb{R}^{3}$ the target 
position at time $t$. In conventional teleoperation, $x_{r e f}$ would typically be treated as the position of the master.

This position tracking error can be quantitatively evaluated across the entire operation time using cross-correlation $(C C)$. This allows to objectively compare the similarity of the actual path $(\bar{x})$ and the desired path $\left(x_{r e f}\right)$ of the slave robots. In the current study, we define this cross-correlation as in (2) to assess the position tracking error across the overall time of maneuvering $(T)$.

$$
C C_{\text {position }}=\frac{\int_{0}^{T} \bar{x}(t) \cdot x_{r e f}(t) d t}{\sqrt{\int_{0}^{T} \bar{x}^{2}(t) d t} \sqrt{\int_{0}^{T} x_{r e f}{ }^{2}(t) d t}}
$$

2) Maneuverability performance: In addition to the conventional approach, maneuverability performance can (and should) take into account the control effort of the operator. From the operator's perspective, it is desirable to achieve equivalent maneuvering accuracy with less control effort. However, the position tracking metric (i.e., $C C_{\text {position }}$ ) does not consider this fact. From this perspective, maneuverability is defined in terms of the ease of the operator in maneuvering the slave robot for achieving accurate tracking performance.

To assess this effect, we propose to consider the frequency response profile of the human operator, that is, the dynamical relationship between the operator's intended-force $\left(f_{h} \in \mathbb{R}^{3}\right)$ as the input, and the position tracking accuracy $\left(C C_{\text {position }}\right)$ as the output

$$
\Phi_{\text {maneuv }}(s):=\frac{C C_{\text {position }}(s)}{f_{h}(s)} .
$$

In this regard, maneuverability increases when $f_{h}$ is reduced and when position tracking $C C_{\text {position }}$ is increased (i.e., the position tracking error is small).

From this, two additional performance metrics can be derived for maneuverability. First, the $\pm 3 \mathrm{~dB}$ bandwidth of $\Phi_{\text {maneuv }}$, denoted as $\omega_{b d}$. Second, the $H_{2}$ norm of $\Phi_{\text {maneuv }}$, denoted as $\left\|\Phi_{\text {maneuv }}\right\|_{2}$ and defined in (4)

$$
\left\|\Phi_{\text {maneuv }}\right\|_{2}=\left\|W_{\text {low }} \frac{C C_{\text {position }}}{f_{h}}\right\|_{2}
$$

where $W_{\text {low }}$ is a low-pass weighting function with a cut-off frequency $\omega_{c}$. The selection of $\omega_{c}$ depends on the specific application of bilateral teleoperation systems. As the normal tremor of a human hand occurs at $8 \sim 12 \mathrm{~Hz}$ [28], $\omega_{c}$ can be less than $8 \mathrm{~Hz}$.

The bandwidth and $H_{2}$ norm are useful properties of the system. Respectively, they denote how well the system will track an input and the degree of sensitivity of system output with respect to its input. Concerning our current evaluation, large values of $\omega_{b d}$ and $\left\|\Phi_{\text {maneuv }}\right\|_{2}$ both indicate high maneuverability performance.

\section{B. Perceptual Sensitivity}

1) Force tracking of master device: The force tracking of the master haptic device is measured in terms of how accurately it can track the forces that are transmitted from slave robot(s). This is, generally, evaluated by estimating the

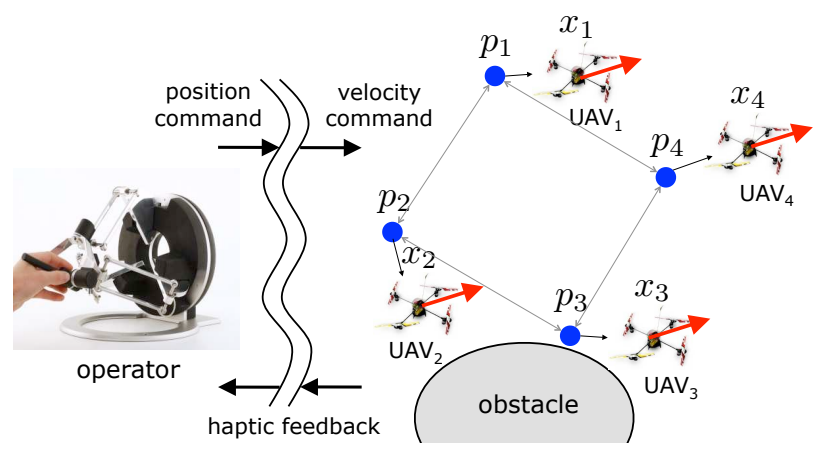

Fig. 1. Haptic teleoperation of multiple UAVs.

discrepancy between the force of the master and the slave. The force tracking error is defined as

$$
e_{f}(t):=f_{m}(t)-f_{\text {ref }}(t)
$$

where $f_{m}(t) \in \mathbb{R}^{3}$ represents the force input of the master device and $f_{\text {ref }}(t) \in \mathbb{R}^{3}$ is the reference force, at time $t$. With conventional teleoperation, $f_{\text {ref }}$ would be replaced by the force exerted on the slaves $\bar{f}_{s}=\frac{1}{N} \sum_{i=1}^{N} f_{s, i}(t), f_{s, i}(t) \in$ $\mathbb{R}^{3}$ represents the force on $i^{t h}$ slave, by being in contact with its immediate environment.

2) Perceptual sensitivity of human operator: Unfortunately, force tracking accuracy is not a sensible measure with regards to the teleoperation of mobile robots (or vehicles, in particular UAVs). As mentioned earlier, UAVs should ideally avoid any direct contact with their environment.

Given that haptic feedback cues should also serve to make human operators sensitive to the remote environment, which is inhabited by the controlled UAVs, it is reasonable to consider sensitivity in terms of their ability to perceptually discriminate between physical differences in the remote environment. For this, it is useful to consider the smallest change in the magnitude of a physical variable (e.g., position, force, and impedance) that can be effectively perceived. Such a measure is commonly referred to as the just noticeable difference (JND) [29]

In this paper, JND is estimated from a psychometric function, which is the probabilistic distribution of a human observer's perceptual response across a chosen physical variable (i.e., distance to an obstacle). For this, the JND is defined as

$$
J N D:=\frac{\Delta f_{r e f}}{f_{r e f}},
$$

which represents the minimum difference in the reference force that can be effectively perceived by the human operator via force feedback.

From our perspective, the JND is more suitable than force tracking accuracy (see (5)) in understanding how human operators can benefit from haptic force feedback in perceiving the remote environment of the multi-UAVs that they control.

\section{Bilateral Teleoperation of Multiple UAVs}

\section{A. Teleoperation Control Architecture}

In [6] we developed a novel control framework which has three control layers: $i$ ) UAV control layer, where each UAV 
is controlled to follow the trajectory of an abstract kinematic virtual point (VP); $i$ ) VP control layer, which modulates each VP's motion according to the teleoperation commands and local artificial potentials; and $i i i$ ) teleoperation layer, through which a remote human user can command the VPs' velocity using a haptic device while haptically perceiving the state of the UAVs over the Internet. Hereafter, we briefly review the control architecture and refer the reader to [6] for further details.

On the master side (see Fig. 1) we consider a 3 degree-offreedoms (DOFs) haptic device modeled by

$$
M(q) \ddot{q}+C(q, \dot{q}) \dot{q}=\tau+f_{h}
$$

where $q \in \mathbb{R}^{3}$ is a configuration of the device (e.g., the position of its end effector), $M(q) \in \mathbb{R}^{3 \times 3}$ is the positivedefinite/symmetric inertia matrix, $C(q, \dot{q}) \in \mathbb{R}^{3 \times 3}$ is the Coriolis matrix, and $\tau, f_{h} \in \mathbb{R}^{3}$ are the control input and human forces, respectively.

The slave side consists of a group of $N$ UAVs, and we denote with $x_{i} \in \mathbb{R}^{3}$ the position of a representative point of the $i$-th UAV, $i=1, \ldots, N$. Define $p_{i}: t \in \mathbb{R} \mapsto p_{i}(t) \in \mathbb{R}^{3}$ as the desired trajectory to be followed by $x_{i}$. We assume that, by adopting a suitable trajectory tracking controller, any smooth reference trajectory $p_{i} \in C^{\infty}$ (VP) can be tracked by the representative point $x_{i}$ with a small error $p_{i}-x_{i}$. This assumption holds, for example, if $x_{i}$ is a flat output [30] for the considered UAV, i.e, if it algebraically defines, with its derivatives, the state and the control inputs of the $i$-th UAV. It is well known that both helicopters and quadrotors satisfy this property [31], [32] ${ }^{1}$.

We denote with $\mathcal{N}_{i}$ the set of UAVs which interact with the $i$-th UAV (commonly denoted as neighbors in the multi-robot literature). The reference trajectory $p_{i}$ is generated online by the kinematic evolution:

$$
\dot{p}_{i}=u_{i}^{c}+u_{i}^{o}+u_{i}^{t} \quad p_{i}(0)=x_{i}(0) .
$$

where the meaning of the three velocity terms $u_{i}^{c}, u_{i}^{o}, u_{i}^{t} \in \mathbb{R}^{3}$ is here briefly explained.

The contribution of $u_{i}^{c} \in \mathbb{R}^{3}$ is aimed at achieving a certain 3D shape formation specified by the desired distances $d_{i j}^{c}, \forall i=1, \ldots, N, \forall j \in \mathcal{N}_{i}$ as well as avoiding a collision among UAVs. In particular $u_{i}^{c} \in \mathbb{R}^{3}$ creates an attractive action if $\left\|p_{i}-p_{j}\right\|$ is large, a repulsive action if $\left\|p_{i}-p_{j}\right\|$ is small, and a null action if $\left\|p_{i}-p_{j}\right\|=d_{i j}^{c}$. Because of the presence of two vertical asymptotes in $u_{i}^{c}$, corresponding to the minimum and the maximum allowed distances, interrobot collisions are guaranteed to be prevented and inter-robot connectivity to be preserved (see [6] for a formal proof). For any obstacle point $p^{o}$ in the environment, the term $u_{i}^{o} \in \mathbb{R}^{3}$ implements a repulsive action if $\left\|p_{i}-p^{o}\right\|$ is small and a null action for $\left\|p_{i}-p^{o}\right\|>D_{o}$ where $D_{o} \in \mathbb{R}^{+}$represents a certain distance threshold. Finally, $u_{i}^{t} \in \mathbb{R}^{3}$ represents a desired velocity term directly controlled by the human operator by means of the position of the master device $q$

$$
u_{i}^{t}=\lambda q \quad \forall i
$$

${ }^{1}$ We refer the interested reader to [33], [34] for the description of some trajectory trackers for the quadrotor case. where $\lambda \in \mathbb{R}^{+}$is a constant scale factor used to match different scales between $q$ and the UAVs desired velocity $u_{i}^{t}$. The desired shape is exactly achieved only if the sum $u_{i}^{o}+u_{i}^{t}$ is the same $\forall i=1, \ldots, N$ (e.g., if $u_{i}^{o}+u_{i}^{t}=0 \forall i$ ), otherwise the resulting group shape is a deformed version of the desired one. In this way the group can automatically adapt to the size of environment (e.g., by shrinking when the operator pushes it in narrow hallways).

\section{B. Haptic Feedback Algorithms}

We take as a starting point, three classes of haptic cues that are typically considered as force feedback sources in conventional teleoperation systems: $i$ ) the mismatch between the command of the master and the execution of the slave in terms of position $q-x_{s}$ or velocity $\dot{q}-\dot{x}_{s}$ (i.e., the masterslave tracking error); $i i$ ) the force measured by a force-sensor mounted on the slave in contact with the environment $f_{s}$; and iii) a linear combination of $i$ ) and $i i$ ) [10]. In the frequency domain, these three cases can be summarized by the following expression for the control input force:

$$
\tau=-C_{p}\left(q-x_{s}\right)-K_{f} f_{s}
$$

where $C_{p}=K_{p}+B_{p} s$ is the PD transfer function of the position controller and $K_{f}$ is the force controller transfer function, being $K_{p}, B_{p}$, and $K_{f}$ diagonal gain matrices [10]. Case $i$ ) corresponds to the situation where $K_{f}=0$ and is commonly referred to as position-position (PP) control or position-error feedback; case $i i$ ) is consistent with the situation where $K_{p}=B_{p}=0$ and is typically referred to as forceposition (FP) control or direct force feedback; finally, case $i i i$ ), where all the gain matrices are non-zero, is usually denoted as position/force-position (PFP) control. Because of practical reasons such as space limitation, safety, and cost, we did not consider the classes of haptic cues that are based on the force measured on the master side, e.g., position-force (PF) and a four channel (4C) control [10], [11].

The design of the force feedback control $\tau$ in (7) originates from these three classes of haptic cues (i.e., PP, FP, and PFP). However, three main adjustments have to be taken into account when transferring these concepts from conventional teleoperation to our case. First, we have several slaves instead of only one, hence the haptic cue in our case must depend on the average of all the UAV contributions. Second, the masterslave tracking error defined above cannot be directly applied given that the desired velocity for the group is proportional to $q$ rather than $\dot{q}$. Therefore, we define the master-slave tracking error in our case as $q-\frac{1}{\lambda N} \sum_{i=1}^{N} \dot{x}_{i}$. Third, there is no real contact force acting on the slave side, therefore we replace $f_{s}$ with the (scaled) average of the velocity terms due to obstacle avoidance $\frac{1}{\lambda N} \sum_{i=1}^{N} u_{i}^{O}$, which plays the role of a repulsing force for the desired trajectory. Note that while the tracking error is directly influenced by the real UAV states (thanks to the presence of $x_{i}$ ), the obstacle force is only a function of the desired trajectory, in particular of $u_{i}^{o}$.

With these considerations in mind we define our force feedback control as

$$
\tau=-B \dot{q}-K y
$$


where $B, K \succ 0$ are diagonal gain matrices, $-B \dot{q}$ represents a damping term whose role is to stabilize the haptic device, and $y \in \mathbb{R}^{3}$ represents a haptic cue informative of the environment surrounding the UAVs and of the UAV motion states. In direct relation with the aforementioned PP, FP, and PFP classes, we consider the following choices for the signal $y$ :

1) Force-cue feedback (Force):

$$
y=y_{\mathrm{F}}:=\frac{1}{\lambda N} \sum_{i=1}^{N} u_{i}^{o} .
$$

This haptic cue is the counterpart of the FP class and plays the role of a repulsive force from the environment w.r.t. the current position of the reference trajectory for the UAV (environmental force). It is only related to the difference between the positions where the UAVs are supposed to go and the location of obstacles. Therefore it is not related to the actual positions of the UAVs or to any real contact of the UAVs with their environment.

2) Velocity-cue feedback (Velocity):

$$
y=y_{\mathrm{V}}:=q-\frac{1}{\lambda N} \sum_{i=1}^{N} \dot{x}_{i} .
$$

This haptic cue corresponds to the PP class and represents the mismatch between the commanded velocity (specified by $q$ in (9)) and the average velocity of real UAVs (tracking-error). The mismatch can be caused by the following reasons: $i$ ) inaccurate model of the UAV, imprecise parameter calibration (e.g., mass of the UAV), or poor quality of the trajectory tracker, $i i)$ external disturbances on the UAV motion (e.g., wind, friction, actual contact with the environment), iii) nonzero velocity terms $u_{i}^{o}+u_{i}^{t}, i=1, \ldots, N$ in the trajectory generator (8), due to the presence of obstacles closer than $D_{o}$ to the group. Therefore, this haptic cue provides the operator more information compared to the previous case. Note that the velocity-cue also includes the Force cue, albeit 'filtered' by the dynamical behavior of the real UAV positions/velocities rather than directly taken from the desired trajectory.

The third and last case is the counterpart of the PFP class and linearly combines the previous two cues:

3) Velocity plus force-cue feedback (Velocity+Force):

$$
y=y_{\mathrm{V}}+y_{\mathrm{F}} .
$$

We end the Section with some final considerations. If the UAVs are maneuvered close to the obstacles, both $y_{\mathrm{V}}$ and $y_{\mathrm{F}}$ are non-zero. In fact, some of the $u_{i}^{o}, i=1, \ldots, N$ will be non-zero, implying that: i) $y_{\mathrm{F}} \neq 0$ by definition and $\left.i i\right)$ the reference trajectory (and consequently the UAVs) are not following exactly the operator commands represented by the term $u_{i}^{t}=\lambda q$. If the velocity term due to the obstacles and the other two terms in (8) are exactly balancing (they sum up to zero) and consequently the UAV are stationary, then $y_{\mathrm{F}} \simeq y_{\mathrm{V}}$. Finally, if the UAV are moving far away from the obstacles in the environment then $y_{\mathrm{F}}=0$ while $y_{\mathrm{V}}$ is in general non-zero because of all the aforementioned reasons (external disturbances, inaccurate calibration, poor trajectory tracker, contact with an unperceived object, etc).

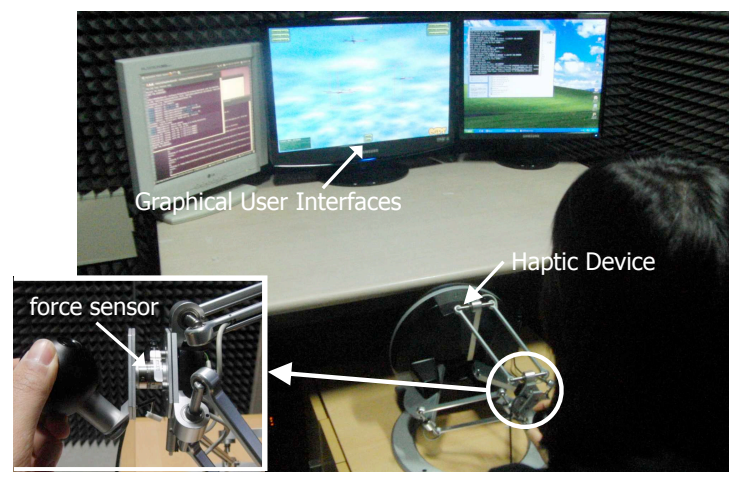

Fig. 2. Experimental setup. Subject with haptic device (Omega 3) and Graphical User Interfaces (GUIs).

\section{General Experimental Methods}

\section{A. Participants}

Thirty-two participants (25 males; age range: $20-33$ years) from Korea University, Seoul were paid approximately $\$ 20$ USD to take part in this study, which consisted of four experiments on maneuverability and perceptual sensitivity for low and large control gains. Five participants took part in all four experiments while the rest took part in at least one experiment. There were eighteen participants for each experiment. All participants possessed normal or corrected-tonormal eyesight and no physical disability. The experiments were conducted in accordance with the requirement of the Helsinki Declaration.

\section{B. Apparatus}

The apparatus mainly consisted of a central display and a haptic device. The former presented a virtual environment for a swarm of four UAVs whose flight path could be controlled by the latter (see Fig. 2).

This multi-UAV swarm always assumed a tetrahedron formation in this virtual environment, with an inter-UAV distance of approximately $0.8 \mathrm{~m}$. The UAVs dynamics and control logic were simulated in a custom-made simulation environment that was based on the Ogre3D engine (for 3D rendering and computational geometry computations), with PhysX libraries to simulate the physical interaction between the UAVs and their virtual environment. This simulation was updated at $60 \mathrm{~Hz}$ which, in turn, constrained the data exchange rate between the haptic device and virtual UAVs. In the display, the simulated UAVs and environment were rendered from a camera perspective that was $32 \mathrm{~m}$ (and $8 \mathrm{~m}$ ) away from the starting positions of the UAVs for the maneuverability (and the perception experiment respectively), with a FOV of about $21^{\circ}$. This rendered scene was presented via the center display monitor.

The UAV swarm was controlled by a commercial haptic device (Omega 3, Force Dimension). The Omega 3 is a 3DOFs haptic device with 3 translational actuated axes and a local control loop running at about $2.5 \mathrm{kHz}$ on a dedicated linux machine. In addition, ATI six-axis force/torque sensors, Nano17, were attached to the Omega 3 device to measure the 
force that the human operator exerted on the device during experimentation, as shown in Fig. 2.

Finally, instructions on manipulating the haptic device and the experimental procedure were presented on the left and right monitors, respectively.

\section{Data Analysis}

Three measures served as performance metrics for maneuverability in our evaluation study, according to our description in Sec. II-A. To recap, $C C_{\text {position }}(\%)$ in (2) represents the similarity of the executed path to the desired path. $\omega_{b d}$ and $\left\|\Phi_{\text {maneuv }}\right\|_{2}$ represents the bandwidth and $H_{2}$ norm of the maneuverability frequency response $\left(\Phi_{\text {maneuv }}\right.$ in (3)). These measures were separately computed for the $\mathrm{x}$-axis and $\mathrm{y}$-axis components of the executed path. In fact, the dynamics of the quad-rotors along $\mathrm{y}$-axis are faster than the $\mathrm{x}$-axis for mechanical reasons. Thus, there were six performance metrics for maneuverability. In our study, $\Phi_{\text {maneuv }}$ was measured as the experimental frequency response of the haptic teleoperation system of multi-UAVs shown in Fig. 2. This considered as input the human operational force to the haptic device $\left(f_{h}\right)$, calculated as

$$
f_{h}(t)=f_{m}(t)-\tau(t)
$$

where $f_{m}$ is the force that was measured from the force sensor on the haptic device and $\tau$ is the master control force given by the current haptic feedback algorithm. The output is $C C_{\text {position }}$ treated in percentage (see (2)). With both variables, it was possible to calculate $\Phi_{\text {maneuv }}$ by applying the empirical transfer function estimator (ETFE [35]), using a second-order low-pass filter ( $W_{\text {low }}$ ) with cut-off frequency of $8 \mathrm{~Hz}$ (see (4)).

Psychometric functions were fitted, in the perception experiment, to the collected data to assess each participant for their discrimination sensitivity, given a particular haptic cue. They were derived using the psignifit toolbox for Matlab which implements the maximum-likelihood method described in [36]. Discrimination sensitivity was measured as the difference of stiffness values between the $16 t h$ and $84 t h$ percentile of the psychometric function. This value was referred to as the JND and denotes the difference in stimulus intensity that participants were able to reliably detect over one standard deviation around the point of subject equality ${ }^{2}$ (PSE). A smaller value indicates greater sensitivity in discrimination; that is, better environmental awareness.

In the maneuverability and perception experiment, all performance metrics were submitted to a one-way repeatedmeasures analysis of variance (ANOVA) for the factor of haptic feedback algorithms (Velocity, Force, and Velocity + Force cues $)^{3}$. An alpha level of 0.05 was taken to indicate statistical significance. When a main effect was found, post-hoc t-tests ${ }^{4}$ were conducted among the haptic feedback algorithm conditions to identify those which were significantly different from each other in terms of the relevant performance metrics.

\footnotetext{
${ }^{2}$ This is value of relative intensity of stimuli for which the test stimuli is perceived to be equaled to the reference stimuli.

${ }^{3}$ Greenhouse-Geisser corrections were applied for instances of unequal variances.

${ }^{4}$ Bonferroni corrected.
}

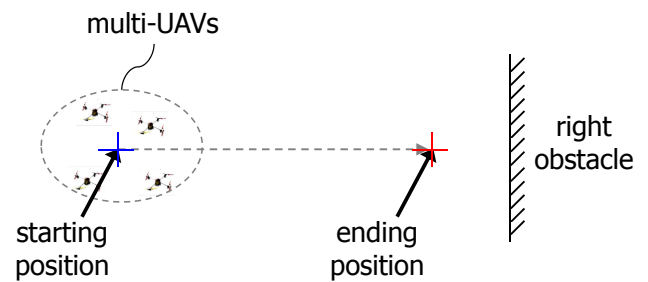

Fig. 3. Sketch of tuning procedure of control parameters
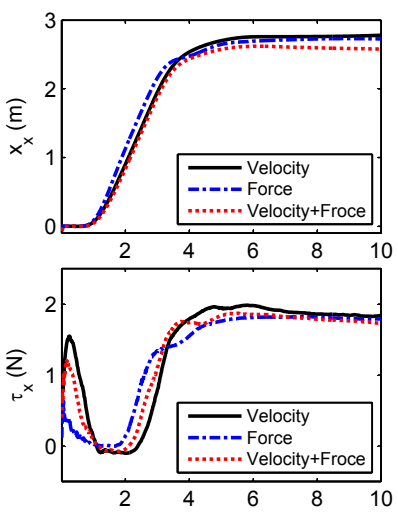

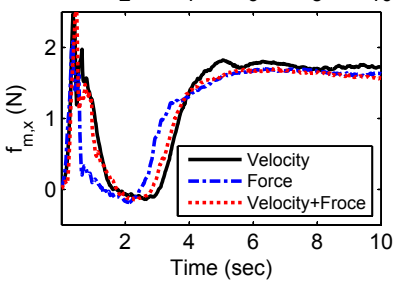

(a)
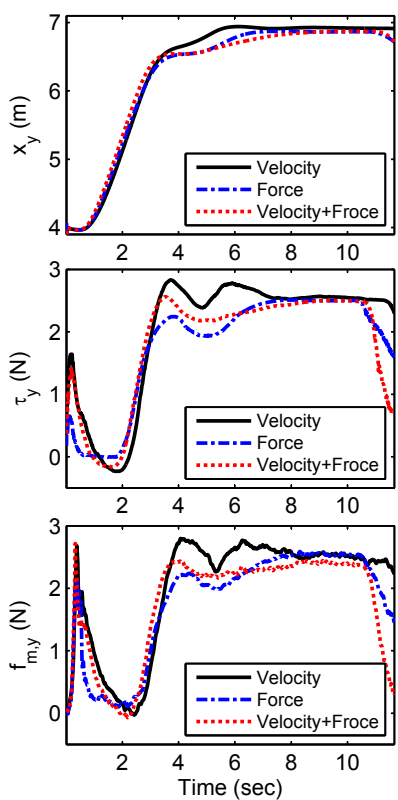

(b)
Fig. 4. Experimental results of control parameters tuning. (a) With right-side obstacle. (b) With upper-side obstacle.

\section{Selection of Control Parameters}

The magnitude of the force feedback is expected to be strongly influenced by the control parameters (gains) $K$ and $B$ which were defined for our force feedback control in (11) with the haptic cueings in (12) (Force cue), (13) (Velocity cue), and (14) (Velocity+Force cue). Here, for a fully fair comparison of the performance of the proposed haptic feedback algorithms the control parameters were tuned to produce a same magnitude of force to the operator.

The multi-UAVs, in a tuning procedure, were maneuvered from a starting position to an ending position located in front of an obstacle as shown in Fig. 3. The UAVs, then, were stopped at the ending position over 5 secs. During this operation $f_{m}$ was measured using the force sensor mounted on the haptic device.

Details of this tuning procedure are reported in the following: first, we fixed the gains as $K=50 \mathrm{~N} / \mathrm{m}$ and $B=2 \mathrm{Ns} / \mathrm{m}$ for the Force cue. Then, keeping this condition as reference, the gains were tuned for the cases of the Velocity and the Velocity +Force cues to produce the same force as in the Force cue case. This tuning procedure was performed in four cases depending on the position of the obstacles relative to the multiUAVs, i.e., obstacles located on the right side, left side, upper side, and down side (see Fig. 3). The values of the tuned 


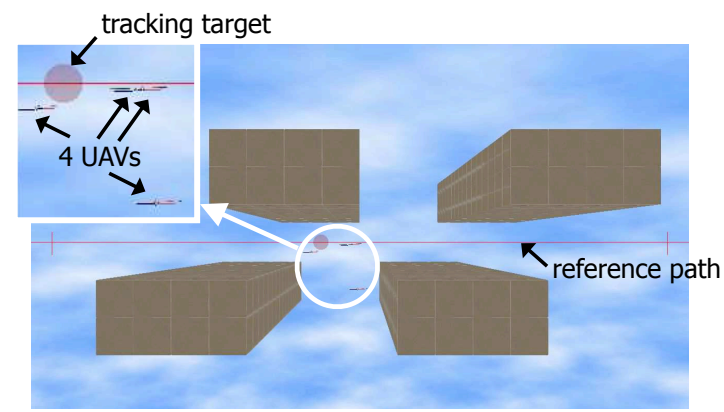

Fig. 5. Screen shot of maneuverability experiment with visible reference path and obstacles, which were rendered invisible during the actual experiment.

control parameters are $K=50 \mathrm{~N} / \mathrm{m}$ and $B=2 \mathrm{Ns} / \mathrm{m}$ for the Velocity cue while $K=30 \mathrm{~N} / \mathrm{m}$ and $B=2 \mathrm{Ns} / \mathrm{m}$ for the Velocity+Force cue. Hereafter, this parameter set will be referred to as low gain. Note that the damping parameter $B$ was not included in the tuning to maintain the same motion agility of the UAVs and the same stability performance based on the passive-set-position-modulation (PSPM) algorithm [37].

Experimental results using the tuned control parameters are shown in Fig. 4. The plots are averages of repeated five experiments. The first row of Fig. 4 shows the trajectories of the center position of the multi-UAVs. The UAVs were operated along the same path with all haptic feedback algorithms. The master control force $\tau$ is shown in the second row. As expected the magnitude of $\tau$ at steady-state keeps similar across the various conditions despite small difference due to transient phases. We can also verify that the operator received very similar forces for all haptic feedback algorithms from the measured force $f_{m}$ shown in the third row of Fig. 4.

Another parameter set was also tested to analyze an effect of control parameters on the system performance. This is defined as $K=70 \mathrm{~N} / \mathrm{m}$ and $B=2 \mathrm{Ns} / \mathrm{m}$ for all Velocity, Force, and Velocity + Force cues and, hereafter, will be referred to as large gain.

\section{EXPERIMENT 1: EVAluAtion OF MANEUVERABility}

The purpose of this experiment was to evaluate how maneuverability performance varied in response to the proposed haptic cue algorithms, in accordance to the proposed performance measures.

\section{A. Procedure}

In this experiment, participants were required to maneuver a swarm of UAVs, using the haptic control device. The objective was always to follow a moving target. This moving target preceded the UAV swarm and moved along a preassigned path, which was either straight or curved (see Fig. 5 and [38] for details). Prior to experimentation, the participants were given a detailed tutorial about the experiment and were provided a training session with the Omega for about 10 mins to get them familiarized with the haptic device and the procedure.

The full experiment was separated into three blocks of 25 trials each. Each block of trials was defined by the design of the haptic cue that provided feedback to the participant; namely, Velocity, Force, and Velocity+Force cues.

On each trial, participants were presented with one of three possible scenarios for UAVs maneuvering; 5, 10 and 10 trials for Scenario 1 (a straight path with no bounding obstacles), for Scenario 2 (a straight path that was bounded by four obstacles) and for Scenario 3 (a curved path which was bounded by four obstacles), respectively. These scenarios were featured in all of the blocks and were fully randomized within the block for their presentation order. It should be noted that the obstacles as well as the reference path were invisible during the experiment to minimize any influence of visual feedback. Only the UAVs and moving targets were visible during the experiment. Finally, the presentation order of the blocks was fully counter-balanced across the participants, to minimize the influence of practice and order effects on our findings.

\section{B. Results}

Figs. 6-8 are summaries of performance in this maneuvering experiment. Performance on the three different scenarios were independently analyzed, for low and large values of gain. The results are reported in the following sub-sections.

1) Scenario 1-straight path with no obstacles: With low gain values, the haptic cue conditions did not significantly differ on any of the performance measures for maneuverability. On the other hand, with large gain values, the Force cue condition yielded significantly larger $\left\|\Phi_{\text {maneuv }}\right\|_{2}$ values compared to the Velocity+Force condition [38].

Direct comparisons between the low and large gain conditions of each haptic cue indicated the following. There was no significant difference on any performance measure for the Velocity cue. For the Force cue, larger gains significantly increased the values of $\left\|\Phi_{\text {maneuv }}\right\|_{2}$ for both the horizontal and vertical components (respectively, $t_{34}=2.55, p<0.05$; $\left.t_{34}=2.23, p<0.05\right)$. In the case of the Velocity + Force cue larger gains significantly decreased the value of $\omega_{b d}$ $\left(t_{34}=2.42, p<0.05\right)$.

2) Scenario 2-straight path with obstacles: As reported in [38], with large gain, the Force cue yielded significantly higher performance compared to Velocity and Velocity+Force cues for both the horizontal and vertical components. With low gain values, however, the haptic cue conditions significantly differed on the measure of $\left\|\Phi_{\text {maneuv }}\right\|_{2}$, for the horizontal component of flight $\left(F_{2,34}=24.3, p<0.001\right)$, but not on the other measures. Specifically, the Force cue yielded significantly higher $\left\|\Phi_{\text {maneuv }}\right\|_{2}$ values, relative to both Velocity and Velocity +Force cues.

Direct comparisons between the low and large gain conditions of each haptic cue indicated the following. There was no significant difference on any performance measure for the Velocity cue. Increasing the gain for the Force cue significantly increased the values of $\left\|\Phi_{\text {maneuv }}\right\|_{2}$ for both the horizontal and vertical components (respectively, $t_{34}=2.73, p<0.05$; $\left.t_{34}=2.35, p<0.05\right)$. In addition, doing so significantly decreased the $\omega_{b d}$ on the vertical component $\left(t_{34}=3.68\right.$, $p<0.01)$. Last, increasing the gain for the Velocity+Force cue significantly decreased the $\omega_{b d}$ on the vertical component $\left(t_{34}=2.22, p<0.05\right)$. 


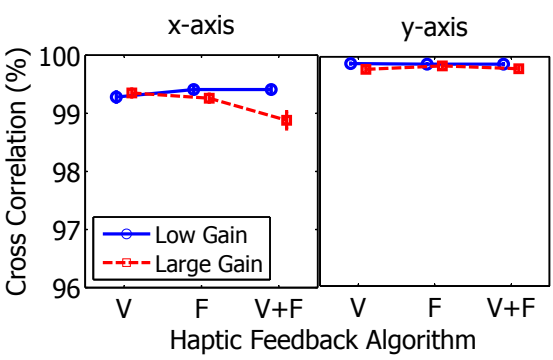

(a)

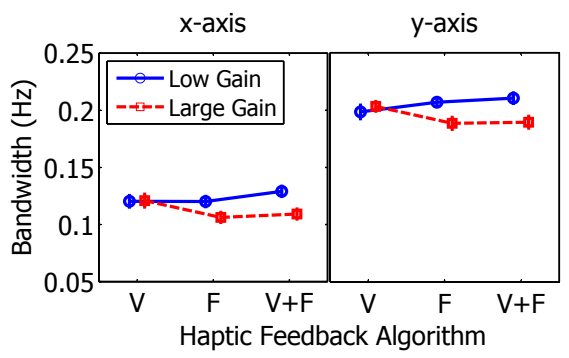

(b)

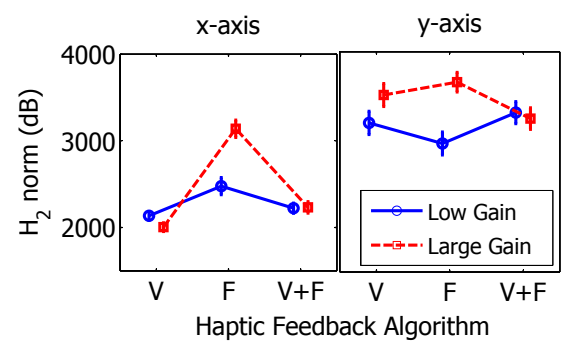

(c)

Fig. 6. Maneuverability performance in the Scenario 1. Figures are plotted as median \pm standard error: (a) Cross correlation of the position tracking, $C C_{\text {position }}(\%)$. (b) Bandwidth of the maneuverability, $\omega_{b d}$. (c) $H_{2}$ norm of the maneuverability, $\left\|\Phi_{\text {maneuv }}\right\|_{2}$.

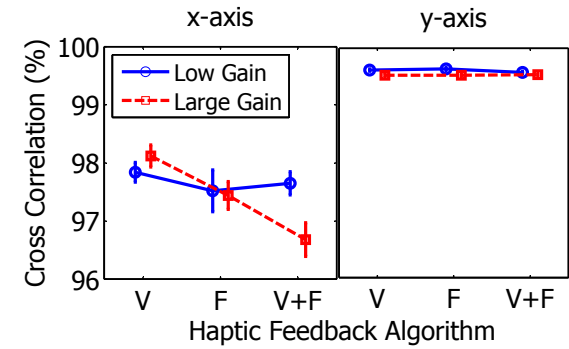

(a)

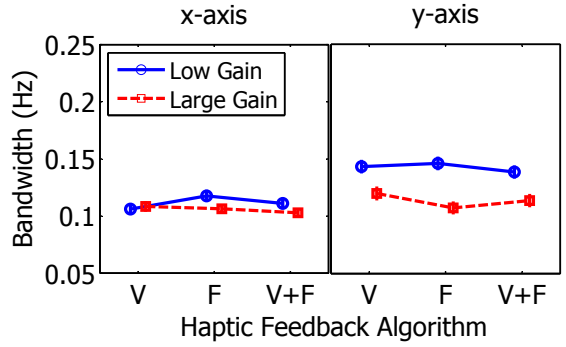

(b)

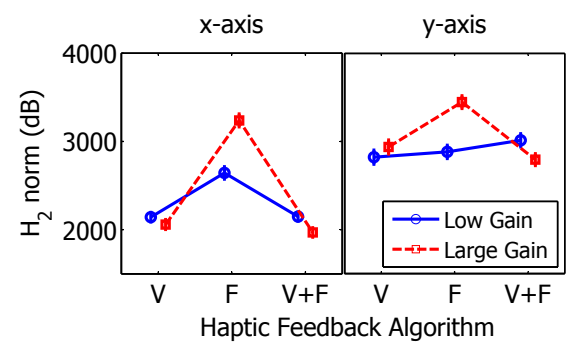

(c)

Fig. 7. Maneuverability performance in the Scenario 2. Figures are plotted as median \pm standard error: (a) $C C_{\text {position }}(\%)$. (b) $\omega_{b d}$. (c) $\left\|\Phi_{m a n e u v}\right\|_{2}$.

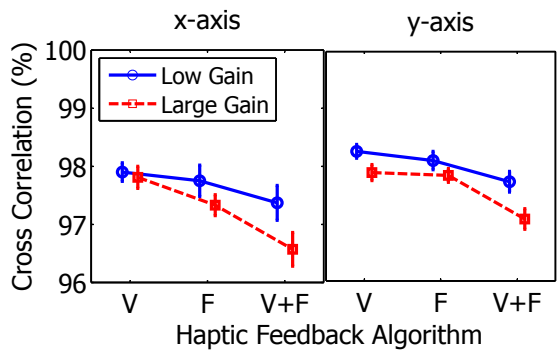

(a)

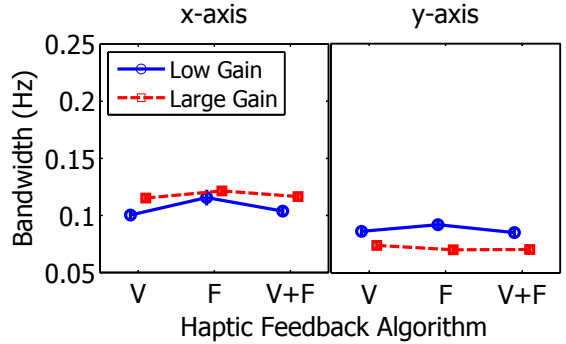

(b)

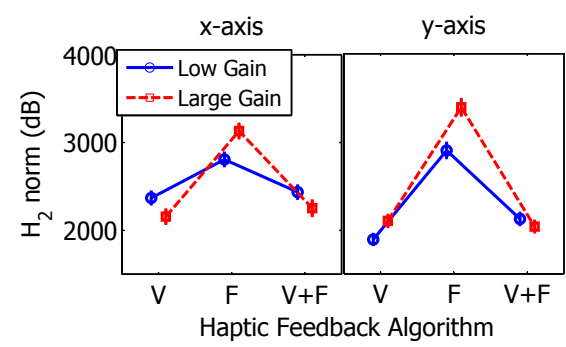

(c)

Fig. 8. Maneuverability performance in the Scenario 3. Figures are plotted as median \pm standard error: (a) $C C_{\text {position }}(\%)$. (b) $\omega_{b d}$. (c) $\left\|\Phi_{m a n e u v}\right\|_{2}$.

3) Scenario 3-curved path with obstacles: With low gain values, the haptic cue conditions significantly differed on the measure of $\left\|\Phi_{\text {maneuv }}\right\|_{2}$, for both the horizontal and vertical component of flight (respectively, $F_{2,34}=6.38, p<0.01$; $F_{2,34}=27.46, p<0.001$ ), but not on the other measures. In both instances, the Force cue yielded significantly higher values compared to both Velocity and Velocity+Force cues. With large gain values, the haptic cue conditions significantly differed on the measures of $C C_{\text {position }}$ and $\left\|\Phi_{\text {maneuv }}\right\|_{2}$. With regards to $C C_{\text {position }}$, the Velocity cue yielded significantly higher values than Velocity+Force cue. However, with regards to $\left\|\Phi_{\text {maneuv }}\right\|_{2}$, the Force cue yielded higher values than both Velocity and Velocity+Force cues.

Direct comparisons between the low and large gain conditions of each haptic cue indicated the following. For the Velocity cue, larger gains induced significantly larger $\omega_{b d}$ on the horizontal component $\left(t_{34}=2.43, p<0.05\right)$ and smaller $\omega_{b d}$ on the vertical component $\left(t_{34}=2.23, p<0.05\right)$. Also, it resulted in significantly larger $\left\|\Phi_{\text {maneuv }}\right\|_{2}$ in the vertical component $\left(t_{34}=2.00, p<0.05\right)$. For the Force cue, larger gains induced significantly larger $\left\|\Phi_{\text {maneuv }}\right\|_{2}$ in the vertical component $\left(t_{34}=3.73, p<0.001\right)$. Last, increasing the gain for the Velocity+Force cue induced significantly reduced $\omega_{b d}$ on the vertical component $\left(t_{34}=2.19, p<0.05\right)$.

\section{Discussion}

In general, the Force cue algorithm for haptic feedback consistently yields better maneuverability performance than the Velocity algorithm as well as the Velocity+Force algorithm. This is demonstrated for the measure of $\left\|\Phi_{\text {maneuv }}\right\|_{2}$ across the three tested scenarios. The results show that the operator required less force to achieve the same level of maneuvering accuracy when the Force cue feedback was implemented. Broadly speaking, this advantage of using the Force cue feedback is more pronounced with the larger values of gain and when obstacles were present. Therefore, Force cue feedback afford greater sensitivity in the transfer from the input force to tracking accuracy. In other words, less control effort is required on the part of the operator in achieving the same level of tracking accuracy with Force cues. 


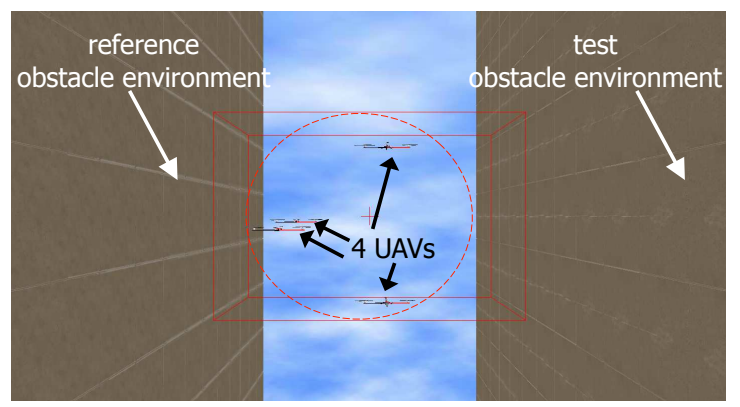

Fig. 9. Screen shot of test of perceptual sensitivity evaluation with visible obstacles, which were rendered invisible during the actual experiment.

With regards to tracking accuracy itself, the measure of $C C_{\text {position }}$ was less sensitive as a performance measure of maneuvering accuracy. It only revealed distinctions between the haptic feedback algorithms with large gain values and only in the presence of obstacles. When significant differences were revealed, the Velocity cue feedback proved to be significantly better than the Velocity+Force cue feedback, but not the Force cue feedback.

Finally, varying gain values tended to influence the bandwidth $\left(\omega_{b d}\right)$ of the frequency response profile. Larger gain values typically corresponded with lower $\omega_{b d}$, especially for the Velocity+Force cue, although it can be apparent on all algorithm types.

\section{EXPERIMENT 2: EVAlUATION OF PERCEPTUAL SENSITIVITY}

The purpose of this experiment was to evaluate how the operator's perceptual sensitivity of obstacles in the multi-UAVs' (remote) environment could be influenced by the proposed haptic cue algorithms.

\section{A. Procedure}

In this experiment, participants were presented with a swarm of four UAVs on each trial, which were located between two invisible obstacles (see Fig. 9). Their task was to move the multi-UAV swarm towards the direction of the obstacles and to determine which of the two obstacles returned a stiffer response on the haptic control device. Participants indicated the stiffer of the two obstacles by using a mouse to click on one of two possible buttons that were located at the left or right position of the screen, which corresponded to the respective positions of the obstacles.

The experiment was divided into three blocks of trials for the three haptic cues. The presentation order of these blocks was counter-balanced across the participants, such as to minimize practice effects.

Throughout the experiment, one obstacle was held at a constant stimulus level (distance, $3.4 \mathrm{~m}$ ) while the other differed from this reference wall across 7 levels of relative stimuli, from $50 \%$ to $150 \%$ in equal steps, by the method of constant stimuli [29]. The positions of the obstacles, relative to each other, were counterbalanced throughout the experiment and there were 10 trials for each level of tested difference, resulting in a total of 70 trials per block. These trials were completely

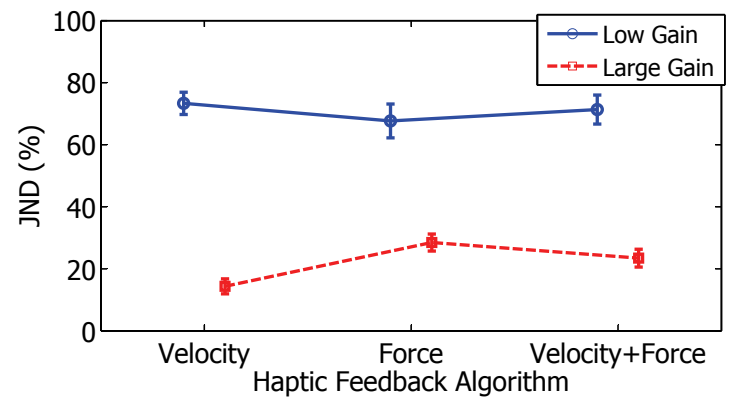

Fig. 10. Summary of JNDs across the haptic cue conditions plotted as median \pm standard error.

randomized for presentation order. Instructions were provided prior to experimentation, as well as a practice session, to ensure good performance during testing like Experiment 1. Each trial took approximately 20 secs to complete and 10 mins breaks were provided between the blocks of trials. The entire experiment took 90 mins to perform.

It is important to reiterate that only the UAVs were visible to the observer. We rendered the obstacles invisible to minimize any possible influence of visual feedback on our participants' discrimination responses like Experiment 1. This allowed us to specifically address the influence of our haptic cue feedback.

\section{B. Results}

Experimental results are summarized in Fig. 10. Smaller JND values indicate greater perceptual sensitivity to the haptic cue condition.

There was no significant main effect of haptic cue condition with low gains $\left(F_{2,34}=0.49, p>0.05\right)$. However, there was a significant main effect of haptic cue condition when larger gains were applied as reported in [39]. Here, participants were significantly more sensitive to haptic feedback forces that were based on the Velocity information of remote UAVs, cues, compared to Force cue feedback.

Direct comparisons between the low and large gain conditions of each haptic cue revealed significant difference for all the haptic conditions (Velocity cue: $t_{34}=13.7, p<0.001$; Force cue: $t_{34}=6.4, p<0.001$; Velocity+Force cue: $\left.t_{34}=8.7, p<0.001\right)$. Thus, larger gains in haptic feedback result in higher perceptual sensitivity, regardless of haptic cue design.

\section{Discussion}

Generally, participants are more sensitive to haptic forces with larger gains. This is to be expected. However, it is important to note that gains should not be indiscriminately increased. In our current scenario, the application of the same gain magnitudes resulted in differential improvements across the three haptic cue designs.

With larger gains, Force cue feedback fared comparably worse to Velocity cue feedback. In this regard, at least, our current findings contradicts conventional scenarios of teleoperation control (e.g., [22]). With conventional teleoperation, it has been argued that force information tends to bring about better perceptual awareness [22]. This, however, may not be 
the case with UAVs wherein force information is not sensed directly and has to be contrived. The current design of obstacle avoidance force $\left(u_{i}^{o}\right.$ in (8)) as well as the connectivity preservation force $\left(u_{i}^{c}\right.$ in (8)) both affect the operator's perception performance. It is clear that this particular design can be further improved in order to bring about better perceptual sensitivity.

Finally, the utility of Velocity+Force cue is mixed across our participants. Some participants appear to be most sensitive to its generated feedback while other participants are least sensitive to it. It is possible that our participants were unable to intuitively interpret haptic feedback that was contrived from two sources of information and while some preferred the Velocity + Force cue which is presumably richer in information, others construed this as being confounding or inconsistent.

\section{General Discussion}

\section{A. Maneuverability}

Force cue results in multi-UAV control that requires less effort. This is because Force cue feedback is absent during free motion. In contrast, the Velocity cue is absent only when UAVs move with a constant velocity vector, which rarely happens. Therefore, the latter case constantly requires the operator to counter-balance the UAVs' felt inertia in order to obtain the desired motion.

The current approach of considering the frequency response profile of the human operator proved to be important in distinguishing between our haptic cue designs for maneuvering performance. Our approach highlighted the specific aspect by which the Force cue differed from its Velocity counterpart. It is important to note that the measure of tracking accuracy, which is the more conventional measure of maneuverability (e.g., [13], [14]) did not discriminate between the haptic cue designs across our experimental scenarios. With this in mind, future studies should consider adopting the current approach in assessing maneuvering performance.

Evaluating maneuvering performance in terms of the operator's frequency response need not be restricted to the force input of the operator's motor command and the output of tracking accuracy. For example, other behavioral inputs that are relevant to maneuvering efficiency can be modeled in lieu of $f_{h}$ (see (15)). This could include measured stress levels or cognitive workload. Besides the human operator, the same approach could provide additional insight into the relationship of the parameters of haptic controllers and desired maneuverability outcome. Similarly, the desired output could be described in terms of other equally desirable objectives besides tracking accuracy (e.g., system stability, velocity and force tracking).

Another point that is worth noting is that our current measurement of $f_{h}$ consists of both the voluntary motor commands of the operator ( $f_{h}^{\text {active }}$ ) as well as forces that result from the operator's involuntary musculoskeletal reflexes, to movements in the haptic device $\left(f_{h}^{\text {response }}\right)$. Although it was not feasible to discriminate between the two in our current study, future work could address this distinction by obtaining direct measurements from the brain (e.g., using the electroencephalography (EEG) [40]) which like $f_{h}^{\text {active }}$ could represent the voluntary input of the operator. Alternatively, $f_{h}^{\text {response }}$ could be estimated from an appropriate dynamic model (e.g., quasi-linear second-order model [41]) of the wrist or elbow joint as well as from direct measurements of the skeletal muscles (e.g., using the electromyography (EMG) [42]). With this, $f_{h}^{a c t i v e}$ could be calculated by discounting the estimated $f_{h}^{\text {response }}$ from the measured $f_{h}$.

Finally, a moving target was used in our current study to explicitly indicate the desired control path. Nonetheless, operators might vary in terms of what they might individually consider to be desirable control paths, especially for increasingly complex paths. Eye-tracking measurements of where the operators are fixating during UAV control could indicate the intended target destination. We intend to address this in a subsequent study and, in doing so, estimate free UAV control in the absence of explicit instruction.

\section{B. Perceptual Sensitivity}

Perceptual awareness of the remote environment via haptic feedback is important, especially for obstacle avoidance. In our current framework, the presence of proximal obstacles is rendered more detectable by haptic feedback that is based on the UAVs' velocity information, relative to force information. This is important to note, especially for instances where visual feedback is degraded or simply unavailable.

Full visual information was provided to our participants throughout the current experiments. In the real world, however, such information are unlikely to be readily available due to the limitations of on-board camera (e.g., restricted FOV, poor camera resolution) [8], [9] or the external disturbances (e.g., dust, smoke, or steam on the camera) [43]. Even if visual information can be effectively captured, the data volume that has to be transmitted will be considerably more than that which is sufficient for fabricated force cues.

With this in mind, the extra effort that is required when Velocity cues are employed for multi-UAVs control might be justifiable in situations that require participants to be highly aware of nearby objects. Such a situation could be when multiUAVs are used to probe the spatial layout of an environment; for example, search-and-rescue scenarios of enclosed spaces.

\section{Selection of Control Parameters}

The current study also investigated how performance benefits of haptic feedback cue related with the control parameter of gain (i.e., $K$ ). The results of the experiments were unequivocal. Large gain values are necessary in order for haptic feedback cues to have a noticeable effect on performance. The positive contribution of the Force cue to maneuvering performance particularly benefited from larger gain values (see Figs. 6(c) - 8(c)). Nonetheless, it would be wrong to assume that larger feedback forces will automatically deliver improved human performance.

In fact, the largest force feedback was quantitatively returned by the Velocity+Force cue with large gains. However, Velocity+Force cue consistently returned poor performance, for the evaluations of both maneuvering and perceptual sensitivity. In addition, increasing gain values can result in a 
decrease in the bandwidth of the frequency response profile of maneuvering performance. In other words, larger gain values can decrease the effectiveness of the system in tracking the operator's control input.

Subsequent research would be necessary in investigating the functional relationship between gain values of haptic force feedback and human performance. This will be useful in determining optimal gain values for the various haptic cue designs.

\section{CONCLUSION}

In this paper, we presented a framework that supports the transmission of haptic cues from the controlled UAVs to the teleoperator's controller. Haptic cues can be designed to increase the teleoperator's maneuvering performance of the UAVs and perception of the remote environment. For this purpose, we proposed three possible types of haptic cues and three complementary teleoperation controllers based on wellknown conventional teleoperation control. These three designs were based on the UAVs' $i$ ) obstacle avoidance force, $i i$ ) velocity information, and iii) a combination of the two.

In addition, we proposed metrics for assessing maneuverability performance, which takes into account the human operator's control effort during bilateral teleoperation. With respect to this, we found $H_{2}$ norm to be a more reliable measure than the more conventional measure of position tracking accuracy (e.g., [13], [14]). More importantly, this allowed for a more comprehensive assessment of maneuvering performance. We were able to determine that the Force cue feedback algorithm resulted in better maneuvering performance, in the sense that teleoperators were able to achieve equivalent steering of the remote UAVs with significantly less control effort. On the other hand, we proposed JND for assessing the operator's perception ability on the environment instead of conventional force tracking accuracy (e.g., [10], [11]). With the JND, the operator's perception is evaluated better than in the conventional way from the human perspective: the perception performance with the three haptic feedback algorithms could be compared by using discrimination tasks and measuring the JND. Contrary to the maneuverability case, the results showed that the Velocity cue based haptic feedback algorithm was the best (statistically significant).

Maneuverability performance as well as perceptual awareness are equally important qualities during bilateral teleoperation. In light of this, the appropriate haptic feedback algorithm ought to be selected according to the application and the objective of the operator. The current evaluation of fabricated haptic cues for their influence on performance should allow us to design better algorithms for controlling multiple UAVs. Given that our control framework allows for the joint control of multiple robots with a single input, we can expect our findings to generalize to the teleoperation of single robots (e.g., mobile robot [9], UAV [44]).

Ultimately, the appeal of this study lies in the fact that it allows performance of the human operator to be quantified in a manner that can be integrated into control systems. This will motivate subsequent research that seeks to enhance the overall performance of control systems from the human perspective with an optimization in design and selection of the system parameters by using the proposed metric and psychophysical evaluation.

\section{REFERENCES}

[1] R. Beckers, O. Holland, and J. Deneubourg, "From local actions to global tasks: Stigmergy and collective robotics," in Artificial life IV, vol. 181, pp. 181-189, 1994.

[2] C. Jones, D. Shell, M. Mataric, and B. Gerkey, "Principled approaches to the design of multi-robot systems," in Proceedings of the Workshop on Networked Robotics, IEEE/RSJ International Conference on Intelligent Robots and Systems, 2004.

[3] K. P. Valavanis and K. P. Valavanis, Advances in Unmanned Aerial Vehicles: State of the Art and the Road to Autonomy. Springer Publishing Company, Incorporated, 2007.

[4] E. Rodriguez-Seda, J. Troy, C. Erignac, P. Murray, D. Stipanovic, and M. Spong, "Bilateral Teleoperation of Multiple Mobile Agents: Coordinated Motion and Collision Avoidance," IEEE Transactions on Control Systems Technology, vol. 18, no. 4, pp. 984-992, 2010.

[5] D. Lee, "Passive decomposition and control of nonholonomic mechanical systems," IEEE Transactions on Robotics, vol. 26, no. 6, pp. 978992, 2010.

[6] D. Lee, A. Franchi, P. Robuffo Giordano, H. I. Son, and H. H. Bülthoff, "Semi-autonomous haptic teleoperation of multiple unmanned aerial vehicles over the internet," in Proceedings of the IEEE International Conference on Robotics and Automation, pp. 1341-1347, May 2011.

[7] A. Franchi, P. Robuffo Giordano, C. Secchi, H. I. Son, and H. H. Bülthoff, "A passivity-based decentralized approach for the bilateral teleoperation of a group of UAVs with switching topology," in Proceedings of the IEEE International Conference on Robotics and Automation, pp. 898-905, May 2011.

[8] J. McCarley and C. Wickens, "Human factors implications of UAVs in the national airspace," Aviation Human Factors Division, Savoy, Illinois, Technical report AHFD-05-05/FAA-05-01, 2005.

[9] N. Diolaiti and C. Melchiorri, "Teleoperation of a mobile robot through haptic feedback," in IEEE International Workshop HAVE, Haptic Virtual Environments and Their Applications, pp. 67-72, 2002.

[10] K. Hashtrudi-Zaad and S. E. Salcudean, "Analysis of control architectures for teleoperation systems with impedance/admittance master and slave manipulators," The International Journal of Robotics Research, vol. 20, no. 6, pp. 419-445, 2001.

[11] D. Lawrence, "Stability and transparency in bilateral teleoperation," IEEE Transactions on Robotics and Automation, vol. 9, no. 5, pp. 624637, 1993.

[12] K. Fite, L. Shao, and M. Goldfarb, "Loop shaping for transparency and stability robustness in bilateral telemanipulation," IEEE Transactions on Robotics and Automation, vol. 20, no. 3, pp. 620-624, 2004.

[13] I. Aliaga, A. Rubio, and E. Sanchez, "Experimental quantitative comparison of different control architectures for master-slave teleoperation," IEEE Transactions on Control Systems Technology, vol. 12, no. 1, pp. 211, 2004.

[14] Y. Yokokohji and T. Yoshikawa, "Bilateral control of master-slave manipulators for ideal kinesthetic coupling-formulation and experiment," IEEE Transactions on Robotics and Automation, vol. 10, no. 5, pp. 605620, 1994

[15] J. Chen, E. Haas, and M. Barnes, "Human performance issues and user interface design for teleoperated robots," IEEE Transactions on Systems, Man, and Cybernetics, Part C: Applications and Reviews, vol. 37, no. 6, pp. 1231-1245, 2007

[16] S. Ito and Y. Yokokohji, "Maneuverability of master control devices considering the musculo-skeletal model of an operator," in Proceedings of the World Haptics Conference, pp. 57-62, 2009.

[17] M. Nambi, W. Provancher, and J. Abbott, "On the Ability of Humans to Apply Controlled Forces to Admittance-Type Devices," Advanced Robotics, vol. 25, no. 5, pp. 629-650, 2011.

[18] M. Çavusoglu, A. Sherman, and F. Tendick, "Design of bilateral teleoperation controllers for haptic exploration and telemanipulation of soft environments," IEEE Transactions on Robotics and Automation, vol. 18 , no. 4, pp. 641-647, 2002.

[19] G. De Gersem, H. Van Brussel, and F. Tendick, "Reliable and enhanced stiffness perception in soft-tissue telemanipulation," The International Journal of Robotics Research, vol. 24, no. 10, pp. 805-822, 2005. 
[20] P. Malysz and S. Sirouspour, "Nonlinear and filtered force/position mappings in bilateral teleoperation with application to enhanced stiffness discrimination," IEEE Transactions on Robotics, vol. 25, no. 5, pp. 1134-1149, 2009

[21] D. Botturi, M. Vicentini, M. Righele, and C. Secchi, "Perception-centric force scaling in bilateral teleoperation," Mechatronics, vol. 20, no. 7, pp. $802-811,2010$.

[22] H. I. Son, T. Bhattacharjee, and H. Hashimoto, "Enhancement in operator's perception of soft tissues and its experimental validation for scaled teleoperation systems," IEEE/ASME Transactions on Mechatronics, no. 6, pp. 1096-1109, 2011.

[23] T. Lam, H. Boschloo, M. Mulder, and M. Van Paassen, "Artificial force field for haptic feedback in UAV teleoperation," IEEE Transactions on Systems, Man and Cybernetics, Part A: Systems and Humans, vol. 39, no. 6, pp. 1316-1330, 2009.

[24] C. Nielsen, M. Goodrich, and R. Ricks, "Ecological interfaces for improving mobile robot teleoperation," IEEE Transactions on Robotics, vol. 23, no. 5, pp. 927-941, 2007.

[25] F. Janabi-Sharifi and I. Hassanzadeh, "Experimental analysis of mobilerobot teleoperation via shared impedance control," IEEE Transactions on Systems, Man, and Cybernetics, Part B: Cybernetics, vol. 41, no. 2, pp. 591-606, 2011.

[26] J. Venrooij, D. Abbink, M. Mulder, and M. van Paassen, "A method to measure the relationship between biodynamic feedthrough and neuromuscular admittance," IEEE Transactions on Systems, Man, and Cybernetics, Part B: Cybernetics, vol. 41, no. 4, pp. 1158-1169, 2011.

[27] D. Abbink, M. Mulder, F. Van der Helm, M. Mulder, and E. Boer, "Measuring neuromuscular control dynamics during car following with continuous haptic feedback," IEEE Transactions on Systems, Man, and Cybernetics, Part B: Cybernetics, vol. 41, no. 5, pp. 1239-1249, 2011.

[28] G. Burdea and M. Akay, Force and touch feedback for virtual reality. John Wiley \& Sons New York, 1996.

[29] G. Gescheider, Psychophysics: the fundamentals. L. Erlbaum Associates, 1997.

[30] M. Fliess, J. Lévine, P. Martin, and P. Rouchon, "Flatness and defect of nonlinear systems: Introductory theory and examples," International Journal of Control, vol. 61, no. 6, pp. 1327-1361, 1995.

[31] M. J. V. Nieuwstadt and R. M. Murray, "Real-time trajectory generation for differentially flat systems," International Journal on Robust and Nonlinear Control, vol. 8, no. 11, pp. 995-1020, 1998.

[32] V. Mistler, A. Benallegue, and N. K. M'Sirdi, "Exact linearization and noninteracting control of a 4 rotors helicopter via dynamic feedback," in IEEE International Symposium on Robots and Human Interactive Communications, pp. 586-593, 2001.

[33] S. Bouabdallah and R. Siegwart, "Backstepping and sliding-mode techniques applied to an indoor micro," in Proceedings of the IEEE International Conference on Robotics and Automation, pp. 2247-2252, 2005.

[34] N. Guenard, T. Hamel, and R. Mahony, "A practical visual servo control for an unmanned aerial vehicle," IEEE Transactions on Robotics, vol. 24, no. 2, pp. 331-340, 2008.

[35] L. Ljung, "On the estimation of transfer functions," Automatica, vol. 21, no. 6, pp. 677-696, 1985.

[36] F. A. Wichmann and N. J. Hill, "The psychometric function: I. fitting, sampling and goodness-of-fit," Perception and Psychophysics, vol. 63, no. 8, pp. 1293-1313, 2001.

[37] D. Lee and K. Huang, "Passive-set-position-modulation framework for interactive robotic systems," IEEE Transactions on Robotics, vol. 26, no. 2, pp. 354-369, 2010.

[38] H. I. Son, L. Chuang, A. Franchi, J. Kim, D. Lee, S.-W. Lee, H. H. Bülthoff, and P. Robuffo Giordano, "Measuring an operator's maneuverability performance in the haptic teleopertion of multiple robots," in Proceedings of the IEEE/RSJ International Conference on Intelligent Robots and Systems, pp. 3039-3046, 2011.

[39] H. I. Son, J. Kim, L. Chuang, A. Franchi, P. Robuffo Giordano, D. Lee, and H. H. Bülthoff, "An evaluation of haptic cues on the tele-operator's perceptual awareness of multiple UAVs' environments," in Proceedings of the IEEE World Haptics Conference, pp. 149-154, June 2011.

[40] J. Millan, F. Renkens, J. Mouriño, and W. Gerstner, "Noninvasive brainactuated control of a mobile robot by human EEG," IEEE Transactions on Biomedical Engineering, vol. 51, no. 6, pp. 1026-1033, 2004.

[41] J. Dolan, M. Friedman, and M. Nagurka, "Dynamic and loaded impedance components in the maintenance of human arm posture," IEEE Transactions on Systems, Man and Cybernetics, vol. 23, no. 3, pp. 698709,1993
[42] E. Clancy, S. Bouchard, and D. Rancourt, "Estimation and application of EMG amplitude during dynamic contractions," IEEE Engineering in Medicine and Biology Magazine, vol. 20, no. 6, pp. 47-54, 2001.

[43] J. Borenstein and Y. Koren, "Teleautonomous guidance for mobile robots," IEEE Transactions on Systems, Man and Cybernetics, vol. 20, no. 6, pp. 1437-1443, 1990.

[44] S. Stramigioli, R. Mahony, and P. Corke, "A novel approach to haptic tele-operation of aerial robot vehicles," in Proceedings of IEEE International Conference on Robotics and Automation, pp. 5302-5308, 2010.

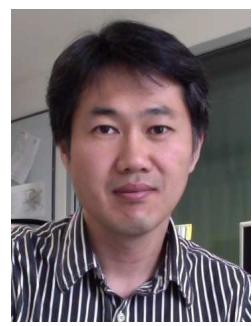

Hyoung Il Son (M'11) received the B.S. and M.S. degrees from the Department of Mechanical Engineering, Pusan National University, Korea, in 1998 and 2000, respectively and the Ph.D. degree from the Department of Mechanical Engineering, Korea Advanced Institute of Science and Technology (KAIST), Korea in 2010

$\mathrm{He}$ is a Research Scientist with the Max Planck Institute for Biological Cybernetics since 2010. He was a senior researcher at LG Electronics (2003-2005) and Samsung Electronics (2005-2009), a research associate at University of Tokyo (2010). His research interests include haptics, teleoperation, human-centered control design and evaluation, and supervisory control of discrete event/hybrid systems.

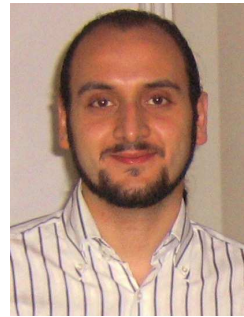

Antonio Franchi (S'07-M'11) received the Laurea degree (summa cum laude) in electronic engineering and the Ph.D. degree in control and system theory from the Sapienza University of Rome, Rome, Italy, in 2005 and 2009, respectively. Since 2010, he has been a Research Scientist with the Max Planck Institute for Biological Cybernetics, Tübingen, Germany. $\mathrm{He}$ was a visiting student with the University of California, Santa Barbara, in 2009. His main research interests include autonomous systems and robotics, with a special regard to planning, control, estimation, humanmachine interaction, haptics, and robotics software architectures.

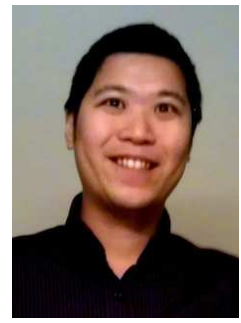

Lewis L. Chuang received the $\mathrm{PhD}$. degree in Neuroscience from the Eberhard-Karl University of Tübingen, Germany, in 2011. He is currently a Postdoctoral Researcher with the Max Planck Institute for Biological Cybernetics, Tübingen, Germany. His research interests lie in understanding how humans actively retrieve and process sensory information in dynamic environments. In doing so, he hopes to inform the development of human-machine interfaces. His research applies a variety of methods, including psychophysics, eyetracking and psychophysiological signals of workload (e.g., heart-rate variability). He is a reviewer for Attention, Perception \& Psychophysics, Vision Research, Journal of Vision, Perception as well as Eurographics.

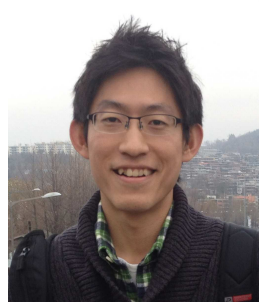

Junsuk Kim received the B.S. degree from the Department of Computer and Radio Communications Engineering and the M.S. degree from the Department of Brain and Cognitive Engineering, Korea University, Korea, in 2010 and 2012, respectively. $\mathrm{He}$ is currently a Ph.D. student in the Department of Brain and Cognitive Engineering, Korea University, Korea. His research interests include psychophysical evaluation of haptic teleoperation and supramodal representations of perceived emotions. 


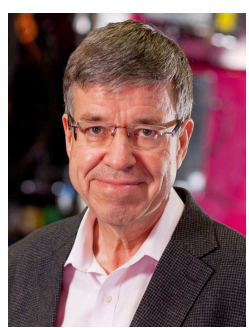

Heinrich H. Bülthoff (M96) received the Ph.D. degree in natural sciences from Eberhard Karls University, Tübingen, Germany, where he received the $\mathrm{Ph} . \mathrm{D}$. degree in biology in 1980 .

From 1980 to 1988 he was a Research Scientist with the Max Planck Institute (MPI) for Biological Cybernetics, Tübingen, and the Massachusetts Institute of Technology, Cambridge. From 1988 to 1993, he was an Assistant, Associate, and Full Professor of cognitive science with Brown University, Providence, RI. He became the Director of the Department for Human Perception, Cognition and Action, MPI for Biological Cybernetics, and a scientific member of the Max Planck Society in 1993. Since 1996, he has been a Honorary Professor with Eberhard Karls University as well as an Adjunct Professor with Korea University, Seoul, Korea. His research interests include object recognition and categorization, perception and action in virtual environments, and humanrobot interaction and perception.

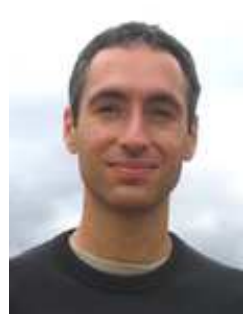

Paolo Robuffo Giordano (M08) received the M.Sc. degree in computer science engineering and the Ph.D. degree in systems engineering from the Sapienza University of Rome, Rome, Italy, in 2001 and 2008, respectively.

From 2007 to 2008, he spent one year as a Postdoctoral Researcher with the Institute of Robotics and Mechatronics, German Aerospace Center. In 2008, he joined the Max Planck Institute for Biological Cybernetics, Tübingen, Germany, where he is currently a Senior Research Scientist Head of the HumanRobot Interaction Group. His research interests include nonlinear control, robotics, haptics, and virtual reality applications. 